\title{
Idiopathic acro-osteolysis: Hajdu Cheney syndrome
}

\section{Asmaa Sqalli Houssaini, Siham Mansouri, Ouiam El Aanzi, Nadia Ismaili, Karima Senouci, Badr Hassam}

Dermatology and venereology department, University Hospital Center Ibn Sina, Rabat, Morocco

Corresponding author: Dr. Asmaa Sqalli Houssaini, E-mail: sqalli_asmae@hotmail.com

\begin{abstract}
Acro-osteolysis (AO) involves partial or total destruction of the distal phalanges of the fingers or toes. The range of forms of $\mathrm{AO}$ is extremely wide. We distinguish acquired forms and the genetic and idiopathic forms. We report a case of 49 years old lady who presented shortening of the fingers on her right hand. We retained an idiopathic AO: Hajdu Cheney syndrome on clinical and radiological criteria as well as the absence of other etiologies. Despite recent improvement in the knowledge of the disease, no cure is available.
\end{abstract}

Key words: Acro-osteolysis; Idiopathic; Hajdu Cheney

\section{INTRODUCTION}

Hajdu-Cheney is a rare, autosomal dominant disorder, with sporadic cases, associated with mutations in the terminal exon 34 of the NOTCH2 gene encoding the transmembrane NOTCH2 receptor and characterized by acroosteolysis of hands and feet, developmental defects of bones, teeth and joints causing distinctive craniofacial and skull changes, and also manifested by severe osteoporosis and short stature [1]. We report a case of Hajdu Cheney syndrome.

\section{CASE REPORT}

A 49 years old lady consulted for a shortening of the fingers on her right hand that had been evolving for several years. The patient had no history of Raynaud's phenomena, occupational exposure to vinyl chloride gas or any recurrent trauma. Clinical examination revealed short stubby fingers with brachyonychia (Fig. 1). Detailed skin and systemic examination failed to detect any abnormalities. A short stature $(1.47 \mathrm{~m})$ was noted. Otherwise, the patient reported that since the age of 15 she had a loss of teeth leading her to put a denture at 30 years.

The radiograph of her right hand showed a complete resorption of the third phalanges (Fig. 2) and the radiograph of the patient's skull revealed an occipital hyperostosis (Fig. 3). Blood counts, hepatic and renal function, Calcium levels, angiotensin converting enzyme and parathyroid hormone were normal. Antinuclear Antibodies (ANA) were negative. Neurological evaluation and The electromyogram were normal. Serological tests for syphilis were negative. The paraneoplastic checkup was normal as well. A diagnosis of idiopathic phalangeal acroosteolysis, Hadju Cheney disease in its sporadic form, was given as it was the most likely judging from the topography of osteolysis, the premature loss of teeth, the occipital hyperostosis, short stature, with no etiological cause identified. Supplementation with calcium and vitamin D. was recommended.

\section{DISCUSSION}

The few primitive forms of $\mathrm{AO}$ are retained after getting rid of medical causes, toxic, traumatic to paraneoplastic.

Radiologically, we consider two types. The longitudinal acro-osteolysis of the distal portion of the phalanx is more common in scleroderma, Raynaud's disease, sarcoidosis, psoriatic arthropathy, neuropathic disease, hyperparathyroidism, porphyria, progeria and

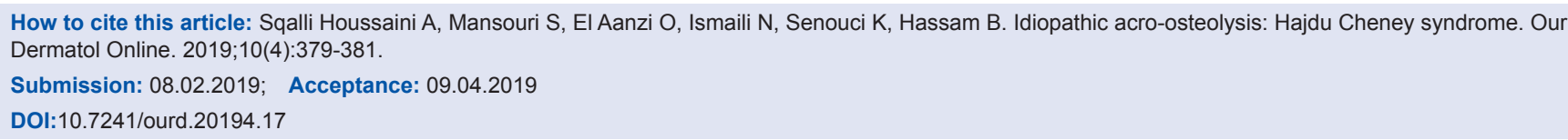




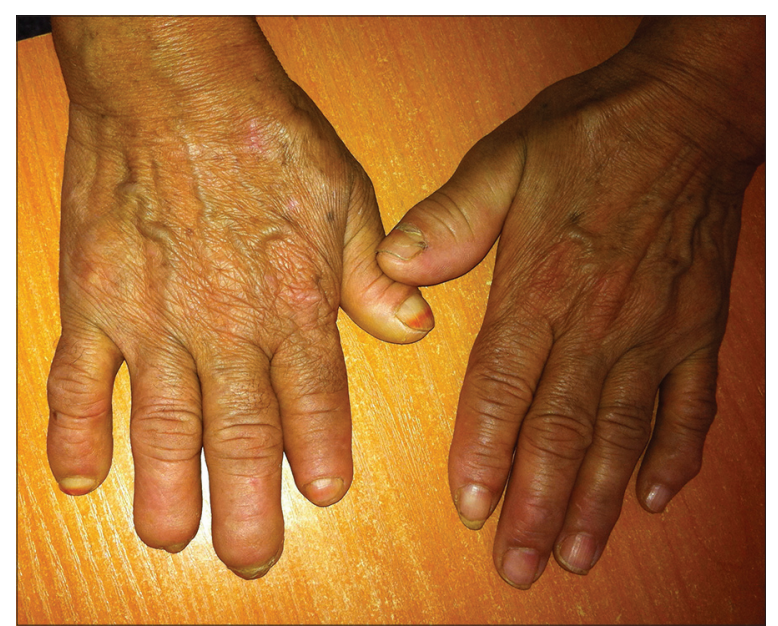

Figure1: Hands showing shortened fingers of the right hand with brachyonychia.

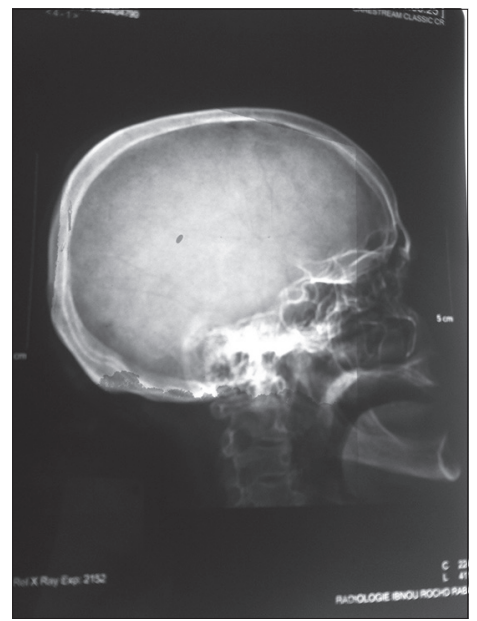

Figure 2: X-rays of the hands showed osteolysis of the $3^{\text {rd }}$ phalanges.

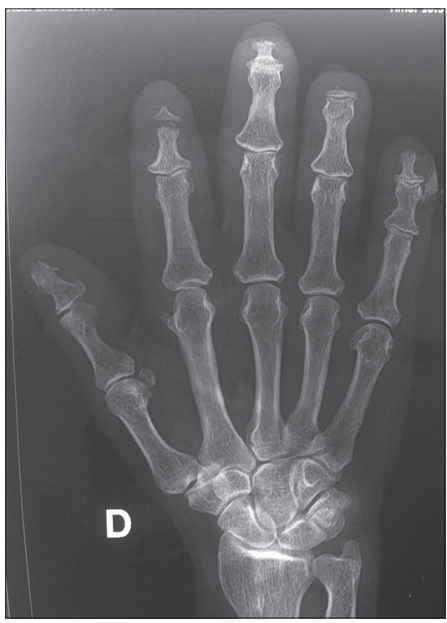

Figure 3: X-rays of the skull: occipital hyperostosis.

pachydermoperiostosis [2] probably secondary to arteritis of inflammatory lesions. The acro-osteolysis, transversally, suggests vinyl chloride poisoning or poisoning due to certain venoms and would be secondary to lesions of vasculitis immunoallergic. It can also appear due to repetitive work that requires constant and repeated pressure on the fingertips.

The idiopathic forms of acro-osteolysis syndrome mainly include the Hajdu Cheney syndrome which is an extremely rare condition (only about 100 recorded cases). Transmission is mainly autosomal dominant, but many cases are sporadic, and evoke new mutations which was the case with our patient who didn't have similar case in her family.

Hajdu Cheney syndrom is reportedly caused by mutations in NOTCH2, which has an important role in the development of the skeleton and in bone remodeling through its action on cells of the osteoblast and osteoclast lineage, and a gain-ofNOTCH2 function results in diverse clinical manifestations. [3]

There are no established criteria for this disease. Clinical manifestations are: acro-osteolysis, severe osteoporosis associated with additional ossification anomalies, hypermobility, and a craniofacial dysmorphic syndrome. AO is generally symmetric and bilateral, and leads to the shorting of digits. Vertebrae are mostly affected with vertebral collapse, which results in a short stature. Dysmorphia involves the loss of teeth, a dolichocephalic skull, a short neck, micrognathia, down-slanted palpepral fissures, hypertelorism, and downturned mouth [4].

Radiographs of patients with Hajdu-Cheney syndrome commonly show acro-osteolysis (band resorption of the middle third distal phalanx), persistent cranial sutures, absent or hypoplastic frontal sinuses, an elongated sella turcica, basilar invagination or platybasia, and biconcave vertebrae. Other clinical features may also be present including kidney cysts and heart defects (coarctation of aorta, patent ductus arteriosus, septal defect), chest deformity with respiratory failure and recurrent respiratory tract infections, ventricular dilatation, basilar compression, syringohydromyelic cavities, hearing loss, and abnormal deep voice. These malformations are rarely observed. In all cases of the literature, reported biological variables are within normal ranges, including phosphocalcic variables [4].

Although the genetic basis of this disease has been deciphered and NOTCH2 has been identified as a causal gene, there is still no cure and patients are currently treated with bone anti-resorptive agents [4] some data suggest that bone mineral density can be 
remarkably improved by bisphosphonate treatment associated with supplementation with calcium and vitamin D $[5,6]$.

\section{CONCLUSION}

Despite recent improvement in the knowledge of the disease, no cure is available. Its evolution is unpredictable; it will either stabilize or extend to the other bones of the hands and feet: hence the interest of long-term monitoring.

\section{REFERENCES}

1. Adami G, Rossini M, Gatti D, Orsolini G, Idolazzi L, et al. Hajdu Cheney syndrome; report of a novel NOTCH2 mutation and treatment with denosumab. Bone. 2016;92:150-156.

2. Datta PK, Ghosh S, De A. Idiopathic non-familial Acro-osteolysis: A rare case report. Indian J Dermatol. 2012;57:486-8.

3. Canalis E, Zanotti S. Hajdu-Cheney syndrome, a disease associated with NOTCH2 mutations. Curr Osteoporos Rep. 2016;14:126-31.

4. Deprouw C, Feydy A, Giraudet Le Quintrec JS, Ruiz B, Kahan A, Allanore Y. A very rare cause of acro-osteolysis: Hajdu-Cheney syndrome. Joint Bone Spine. Joint Bone Spine. 2015;82:455-9.

5. Drake WM, Hiorns MP, Kendler DL. Hadju-Cheney syndrome: response to therapy with bisphosphonates in two patients. J Bone Miner Res. 2003;18:131-3.

6. McKiernan FE. Integrated anti-remodeling and anabolic therapy for the osteoporosis of Hajdu-Cheney syndrome: 2-year follow-up. Osteoporos Int. 2008;19:379-80.

Copyright by Asmaa Sqalli Houssaini, et al. This is an open-access article distributed under the terms of the Creative Commons Attribution License, which permits unrestricted use, distribution, and reproduction in any medium, provided the original author and source are credited.

Source of Support: Nil, Conflict of Interest: None declared. 